\title{
Paired Comparison Analysis of the van Baaren Model Using Bayesian Approach with Noninformative Prior
}

\author{
Saima Altaf \\ Department of Statistics \\ PMAS University of Arid Agriculture, Rawalpindi, Pakistan \\ nodupk@yahoo.com \\ Muhammad Aslam \\ Department of Statistics \\ Quaid-i-Azam University, Islamabad, Pakistan \\ aslamsdqu@yahoo.com \\ Muhammad Aslam \\ Department of Statistics \\ Bahauddin Zakariya University, Multan, Pakistan \\ aslamasadi@bzu.edu.pk
}

\begin{abstract}
One technique being commonly studied these days because of its attractive applications for the comparison of several objects is the method of paired comparisons. This technique permits the ranking of the objects by means of a score, which reflects the merit of the items on a linear scale. The present study is concerned with the Bayesian analysis of a paired comparison model, namely the van Baaren model VI using noninformative uniform prior. For this purpose, the joint posterior distribution for the parameters of the model, their marginal distributions, posterior estimates (means and modes), the posterior probabilities for comparing the two treatment parameters and the predictive probabilities are obtained.
\end{abstract}

Keywords: Bayesian hypothesis testing, Noninformative priors, Posterior distribution, Predictive probability.

\section{Introduction}

When the objects that can be scored on the same scale are compared, they are ranked on the basis of the scores. In some cases, however, it is not possible to assign the score to every object on the same scale, in such cases the objects can only be compared pair wise. In the method of paired comparison, the treatments are presented in pairs to one or more judges who in the simplest situation, choose one from the pair or simply just have no preference.

It was Thurstone (1927), who utilized the technique to make the pairwise comparisons of 19 crimes or offences to compare their seriousness. Zermelo (1929) in his paper proposed a model, which estimated the strengths of the chess players in an uncompleted round robin tournament. Since then wherever sensory testing is involved, the paired comparison technique has found its frequent applications. 
Later on, Bradley and Terry (1952), Bradley (1953), Luce (1959), Rao and Kupper (1967), Davidson (1970) developed and modified the paired comparison models and also considered the possibility of the occurrence of tie when two treatments are being compared.

Beaver and Gokhale (1975), Davidson and Beaver (1977), extended the BradleyTerry and Rao-Kupper models to accommodate the effect of order of presentation of objects in a pair comparison experiment. Van Baaren (1978) also introduced the order effect parameter to a series of paired comparison models in which ties were also accommodated. He presented six different paired comparison models.

The paired comparison method is commonly used in various fields of life e.g. in taste testing experiments, in professional and intercollegiate sports competitions, market research, voting systems, social and public choice, product comparisons performed by the consumers, multidimensional scaling in personal ratings and generally where the study of choice behavior is concerned.

League competitions and round robin tournaments provide good examples of the situations where the rankings are based on the performances of the objects when they meet in pairs. Stern (1990) proposed the method of gamma-paired comparison and applies the model to sports data set for the 1986 National League baseball season.

This technique also has worked surprisingly well for the environmental and ecosystem issues. Neuman and Watson (1993) discuss a study conducted by the Canadian Department of Forestry to determine the relative importance which the Canadians give to the various advantages of the forests. Different benefits obtained by the forests are presented to the respondents in the form of pairs to indicate which value is considered to be more important.

Chess competitions also provide another example. Henry (1992) describes the use of the modified Thurstone-Mosteller model to fit the data based on the results of all the known games of chess played between the past and present world's greatest chess players to find out the greatest chess player. Following the use of paired comparison methods in sports competitions, Marcus (2000) rates the playing strength of the table tennis players by using the Bradley-Terry model.

Paired comparison techniques have also served beneficially in the road safety problems and the quantification of motor vehicle driver's crash risks. Li and Kim (2000) apply the extended Bradley-Terry model for paired comparison to estimate the motor vehicle crash risks using only the crash data.

Space science rests heavily on the visual communications with different space objects and aircrafts etc. therefore, the purpose is always to maximize the visual quality. Watson (2001) proposes the method of paired comparison when the selection is between two physical stimulus intensities in a psychophysical experiment for this purpose. 
Baker et al. (2004) use the paired comparison technique with order effect testing as a tool in the field of sound quality engineering. Judges are asked to choose between pairs of sounds to tell which the most powerful one is

Selection of the students in educational institutes and universities is a very crucial job. Marrin et al. (2004) carry out a survey to identify the tools used to modify the selection procedure for the undergraduate medical program at McMaster University, Canada. Paired comparison methodology is adopted to find out which quality is considered to be most the important which serves as an effective technique in this respect.

Following the application of paired comparison technique in the field of environment and earth sciences, Simons et al. (2006) use this technique to study and compare the communities of breeding birds. Mazzuchi et al. (2008) use Bradley-Terry model for paired comparison for expert judgment to assess the risk of wire failure in aircrafts.

Because of its practical nature and simple use, many of the statisticians became considerate towards this technique and performed Bayesian analyses of the paired comparison models and studied this technique in detail with varying perceptions. These statisticians include Davidson and Solomon (1973), Kim and Kim (2004), Kim (2005), Aslam (2002, 2003, and 2005) and Glickman (2008).

In the current study, the Bayesian analysis of van Baaren model VI is presented. Section 2 is about the notations and likelihood of the model. Section 3 deals with the Bayesian analysis of the model using uniform prior. In Section 4, the appropriateness of the model is tested while Section 5 is reserved for conclusion.

\section{The Van Baaren Model VI for Paired Comparison}

Van Baaren (1978) proposes and compares six extensions of the Bradley-Terry (1952) paired comparison model.

According to Model I, the probability that the preference of $T_{i}$ over $T_{j}$ when $T_{i}$ is presented first is denoted by $\psi_{i j}(1)$ and is presented as:

$$
\psi_{i j}(1)=\frac{\gamma \theta_{i}}{\gamma \theta_{i}+\theta_{j}+v}
$$

where $\gamma(>0)$ is the multiplicative order effect parameter, $v(>0)$ is the tie parameter and $0<\theta_{i}<1$. The preference probability of $T_{j}$ when $T_{i}$ is presented first is denoted by $\psi_{i j}(2)$ and is defined as:

$$
\psi_{i j}(2)=\frac{\theta_{j}}{\gamma \theta_{i}+\theta_{j}+v}
$$


The probability for no preference, which is proportional to the product of both the treatment parameters, is given by:

$$
\psi_{i j}(0)=\frac{v}{\gamma \theta_{i}+\theta_{j}+v}
$$

Model II of van Baaren is given as:

$$
\psi_{i j}(1)=\frac{\gamma \theta_{i}}{\gamma \theta_{i}+\theta_{j}+v \sqrt{\theta_{i} \theta_{j}}}, \psi_{i j}(2)=\frac{\theta_{2}}{\gamma \theta_{i}+\theta_{j}+v \sqrt{\theta_{i} \theta_{j}}}, \psi_{i j}(0)=\frac{v \sqrt{\theta_{i} \theta_{j}}}{\gamma \theta_{i}+\theta_{j}+v \sqrt{\theta_{i} \theta_{j}}}
$$

Van Baaren Model III is given as:

$$
\psi_{i j}(1)=\frac{\gamma \theta_{i}}{\gamma \theta_{i}+v \theta_{j}}, \psi_{i j}(2)=\frac{\theta_{j}}{\gamma v \theta_{i}+\theta_{j}}, \psi_{i j}(0)=\frac{\gamma \theta_{i} \theta_{j}\left(v^{2}-1\right)}{\left(\gamma \theta_{i}+v \theta_{j}\right)\left(\gamma v \theta_{i}+\theta_{j}\right)}
$$

Van Baaren Model IV is described as:

$$
\begin{aligned}
& \qquad \psi_{i j}(1)=\frac{\gamma \theta_{i}}{\gamma \theta_{i}+\theta_{j}+v\left(1+t \sqrt{\gamma \theta_{i} \theta_{j}}\right)}, \psi_{i j}(2)=\frac{\theta_{j}}{\gamma \theta_{i}+\theta_{j}+v\left(1+t \sqrt{\gamma \theta_{i} \theta_{j}}\right)} \text { and for no } \\
& \text { preference } \psi_{i j}(0)=\frac{v\left(1+t \sqrt{\gamma \theta_{i} \theta_{j}}\right)}{\gamma \theta_{i}+\theta_{j}+v\left(1+t \sqrt{\gamma \theta_{i} \theta_{j}}\right)}
\end{aligned}
$$

where $t$ represents the number of objects. Van Baaren Model $\mathrm{V}$ is presented as:

preference

$$
\psi_{i j}(1)=\frac{\gamma \theta_{i}}{\gamma \theta_{i}+\theta_{j}+v \sqrt{\theta_{j} / \gamma \theta_{i}}}, \quad \psi_{i j}(2)=\frac{\theta_{j}}{\gamma \theta_{i}+\theta_{j}+v \sqrt{\gamma \theta_{i} / \theta_{j}}} \quad \text { and } \quad \text { for } \mathrm{no}
$$

$$
\psi_{i j}(0)=\frac{v\left(v+2 \sqrt{\gamma \theta_{i} \theta_{j}}\right.}{\left(\gamma \theta_{i}+\theta_{j}+v \sqrt{\theta_{j} / \gamma \theta_{i}}\right)\left(\gamma \theta_{i}+\theta_{j}+v \sqrt{\gamma \theta_{i} / \theta_{j}}\right)}
$$

And finally according to the van Baaren Model VI, the probability that the preference of $T_{i}$ over $T_{j}$ when $T_{i}$ is presented first is denoted by $\psi_{i j}(1)$ and is defined as:

$$
\psi_{i j}(1)=\frac{\gamma \theta_{i}}{\gamma \theta_{i}+\theta_{j}+v \theta_{i} \theta_{j}}
$$

where $\gamma(>0)$ is the multiplicative order effect parameter, $v(>0)$ is the tie parameter and $0<\theta_{i}<1$. The preference probability of $T_{j}$ when $T_{i}$ is presented first is denoted by $\psi_{i j}(2)$ and is defined as:

$$
\psi_{i j}(2)=\frac{\theta_{j}}{\gamma \theta_{i}+\theta_{j}+v \theta_{i} \theta_{j}}
$$

The probability for no preference, which is proportional to the product of both the preference parameters:

$$
P(i \approx j \mid i, j)=\psi_{i j}(0)=\frac{v \theta_{i} \theta_{j}}{\gamma \theta_{i}+\theta_{j}+v \theta_{i} \theta_{j}}, 0<\theta_{i}<1, \gamma, v>0 .
$$


According to van Baaren, all the six extensions differ only in the way the ties are treated if the order effect parameter is kept constant. In model I, the probability of the tie is the same for all the preference parameters whose sum is constant. This property makes the model far from being realistic. For models II and III, the probability of a tie is the same for all the pairs of preference parameters for which the ratio, the larger over the smaller say is constant. In models IV, V and VI, the probability of a tie depends in addition to the ratio of the preference parameters, on their actual level also.

In addition to this, models I, IV and $\mathrm{V}$ are considered to fit better when the comparison are being made by visual means. On the other hand, model VI is considered to fit best in case of tournaments and matches such as chess tournaments. Furthermore, the model is simpler and easy to handle as compared to III, IV and V models. Keeping this in view, we choose model VI for the Bayesian analysis.

Now as we are considering the order effect parameter also, it means we consider the situation when the order of the two treatments is reversed. Hence, the probability that the preference of $T_{j}$ over $T_{i}$ when $T_{j}$ is presented first is denoted by $\psi_{j i}(1)$ and is defined as:

$$
\psi_{j i}(1)=\frac{\gamma \theta_{j}}{\theta_{i}+\gamma \theta_{j}+v \theta_{i} \theta_{j}}
$$

The preference probability of $T_{i}$ when $T_{j}$ is presented first is denoted by $\psi_{i j}(2)$ and is given as:

$$
\psi_{j i}(2)=\frac{\theta_{i}}{\theta_{i}+\gamma \theta_{j}+v \theta_{i} \theta_{j}}
$$

and hence, for no preference when $T_{j}$ is presented first, the probability is presented as:

$$
\psi_{j i}(0)=\frac{v \theta_{i} \theta_{j}}{\theta_{i}+\gamma \theta_{j}+v \theta_{i} \theta_{j}}
$$

We define notations for the van Baaren model VI; $w_{i j k}(1)=1$ or 0 , accordingly as the treatment $T_{i}$ is preferred to the treatment $T_{j}$ when the treatment $T_{i}$ is presented first in the $k^{\prime}$ th repetition of the comparison.

$w_{i j k}(2)=1$ or 0 , accordingly as the treatment $T_{j}$ is preferred to the treatment $T_{i}$ when the treatment $T_{i}$ is presented first in the $k$ 'th repetition of the comparison. 
$w_{j i k}(1)=1$ or 0 , accordingly as the treatment $T_{j}$ is preferred to the treatment $T_{i}$ when the treatment $T_{j}$ is presented first in the $k^{\prime}$ th repetition of the comparison.

$w_{j i k}(2)=1$ or 0 , accordingly as the treatment $T_{i}$ is preferred to the treatment $T_{j}$ when the treatment $T_{j}$ is presented first in the $k^{\prime}$ th repetition of the comparison.

$t_{i j k}=1$ or 0 , accordingly as the treatment $T_{i}$ is tied with the treatment $T_{j}$ when $T_{i}$ is presented first in the $k$ 'th repetition.

$t_{j i k}=1$ or 0 , accordingly as the treatment $T_{i}$ is tied with the treatment $T_{j}$ when $T_{j}$ is presented first in the $k^{\prime}$ th repetition.

The total number of independent comparisons for the pair $(i, j)$ is given by $r_{i j}$. Let $w_{i j}(1)$ is the number of preferences for the object presented first, $w_{i j}(2)$ be the number of preferences for the object presented second (object $j$ ) and $t_{i j}$ are the number of no preferences, $r_{i j}=w_{i j}(1)+w_{i j}(2)+t_{i j}$ and $r_{j i}=w_{j i}(1)+w_{j i}(2)+t_{j i}$, when the order of presentation is $(j, i)$.

The probability of the observed result in the $k^{\text {th }}$ repetition of the pair of treatments $\left(T_{i}, T_{j}\right)$ when both the orders of presentation i.e. $(i, j)$ and $(j, i)$ are being considered is:

$$
\begin{aligned}
P_{i j k}= & {\left[\frac{\left(\gamma \theta_{i}\right)}{\left(\gamma \theta_{i}+\theta_{j}+v \theta_{i} \theta_{j}\right)}\right]^{w_{j j k}(1)}\left[\frac{\theta_{j}}{\left(\gamma \theta_{i}+\theta_{j}+v \theta_{i} \theta_{j}\right)}\right]^{w_{j j k}(2)}\left[\frac{\left(v \theta_{i} \theta_{j}\right)}{\left(\gamma \theta_{i}+\theta_{j}+v \theta_{i} \theta_{j}\right)}\right]^{t_{j j k}} } \\
P_{i j k} & =\frac{\left(\gamma \theta_{i}\right)^{w_{j j k}(1)} \theta_{j}^{w_{j j k}(2)}\left(\gamma \theta_{j}\right)^{w_{j k k}(1)} \theta_{i}^{w_{j i k}(1)}\left(v \theta_{i} \theta_{j}\right)^{t_{j j k}+t_{j k k}}}{\left(\gamma \theta_{i}+\theta_{j}+v \theta_{i} \theta_{j}\right)^{r_{j j k}}\left(\gamma \theta_{i}+\theta_{j}+v \theta_{i} \theta_{j}\right)^{r_{j i k}}}
\end{aligned}
$$

\section{Bayesian Analysis using Uniform Prior}

Laplace (1812) proposes the Bayesian analysis of the unknown parameters using a uniform prior. For the analysis using noninformative prior, we assume the standard uniform distribution to be the noninformative prior for the parameters of the van Baaren model VI. Let $\theta=\left(\theta_{1}, \ldots, \theta_{m}\right)$ be the vector of treatment parameters, the order effect parameter $\gamma$ and the tie parameter $v$, the uniform prior distribution for the parameters is given as:

$$
p\left(\theta_{1}, \theta_{2}, \ldots, \theta_{m} ; \gamma, v\right) \propto 1 \quad ; \theta_{i} \geq 0, i=1,2, \ldots, m, \sum_{i=1}^{m} \theta_{i}=1, \gamma, v>0 .
$$


The likelihood function of the van Baaren model VI is given as: $l\left(\mathbf{x} ; \theta_{1}, \ldots, \theta_{m}, \gamma, v\right)=\prod_{i \neq j=1}^{m} \prod_{j=1}^{m} P_{i j k}=\prod_{i \neq j}^{m} \prod_{j} \frac{r_{i j} !}{w_{i j}(1) ! w_{i j}(2) ! t_{i j} !}\left[\frac{\left(\theta_{i}^{g_{i}} \gamma^{K} v^{T}\right)}{\left(\gamma \theta_{i}+\theta_{j}+v \theta_{i} \theta_{j}\right)^{r_{i j}}}\right]$,

$$
0<\theta_{i}<1 \text { for } i=1,2, \ldots, m . \gamma, v>0,
$$

where $r_{i j}=w_{i j}(1)+w_{i j}(2)+t_{i j}$. The total number of preferences for the object presented first is $K=\sum_{i=1}^{m} \sum_{j \neq i}^{m} w_{i j}(1)$, the total numbers of ties are $T=\sum_{i=1}^{m} \sum_{j \neq i}^{m} t_{i j}$, $g_{i}=w_{i}+t_{i}, w_{i}=\sum_{j}\left\{w_{i j}(1)+w_{j i}(2)\right\}$ is the total number of wins and $t_{i}=\sum_{j}\left\{t_{i j}+t_{j i}\right\}$ is the total number of ties for the $i^{\text {th }}$ object.

The joint posterior distribution for the parameters $\theta_{1}, \ldots, \theta_{m}, \gamma$ and $v$ using uniform prior is:

$$
\begin{aligned}
& p\left(\theta_{i}, \gamma, v \mid \mathbf{x}\right) \propto \prod_{i \neq j=1}^{m} \prod_{j=1}^{m} \frac{\theta_{i}^{g_{i}} \gamma^{K} v^{\mathrm{T}}}{\left(\gamma \theta_{i}+\theta_{j}+v \theta_{i} \theta_{j}\right)^{r_{i j}}}, \\
& \theta_{i} \geq 0, i=1,2, \ldots, m, \sum_{i=1}^{m} \theta_{i}=1, \gamma, v>0 .
\end{aligned}
$$

The marginal posterior distribution for $\theta_{1}$ is:

$$
\begin{array}{r}
p\left(\theta_{1} \mid \mathbf{x}\right)=\frac{\theta_{1}^{g_{1}}}{Q} \int_{\theta_{2}=0}^{1-\theta_{1}} \ldots \int_{\theta_{m-1}=0}^{1-\theta_{1}-\ldots-\theta_{m-2}} \int_{\gamma=0}^{\infty} \int_{v=0}^{\infty} \prod_{i \neq j=1}^{m-1} \prod_{j}^{m-1} \frac{\theta_{i}^{g_{i}} \gamma^{K} v^{\mathrm{T}}}{\left(\gamma \theta_{i}+\theta_{j}+v \theta_{i} \theta_{j}\right)^{r_{i j}}} d v d \gamma d \theta_{m-1} \ldots d \theta_{2}, \\
0 \leq \theta_{1} \leq 1, \gamma, v>0,
\end{array}
$$

where $Q$ is the normalizing constant. The marginal densities of other parameters can be obtained using the same general form (3.2). The multiple integrals are solved with the help of quadrature method. More complicated integrals are solved with the help of Gibbs sampling method. All the programs are run in the SAS package (programs can be provided by the author if required).

The data for the analysis is taken from Davidson and Beaver (1977) given in Table 3.1 which is of packaged food mixes, with different number of comparisons for each pair.

Table 3.1: Responses for the Preference Testing Experiment for $\boldsymbol{m}=\mathbf{3}$

\begin{tabular}{ccccc}
\hline Pairs $(i, j)$ & $r_{i j}$ & $w_{i j}(1)$ & $w_{i j}(2)$ & $t_{i j}$ \\
\hline$(1,2)$ & 42 & 23 & 11 & 8 \\
$(2,1)$ & 43 & 29 & 6 & 8 \\
$(1,3)$ & 43 & 27 & 11 & 5 \\
$(3,1)$ & 42 & 22 & 14 & 6 \\
$(2,3)$ & 41 & 34 & 6 & 1 \\
$(3,2)$ & 42 & 23 & 16 & 3 \\
\hline
\end{tabular}


The graphs of the marginal densities of the parameters are shown in Fig. 3.1. The curves of all the marginal distributions show symmetric behavior.
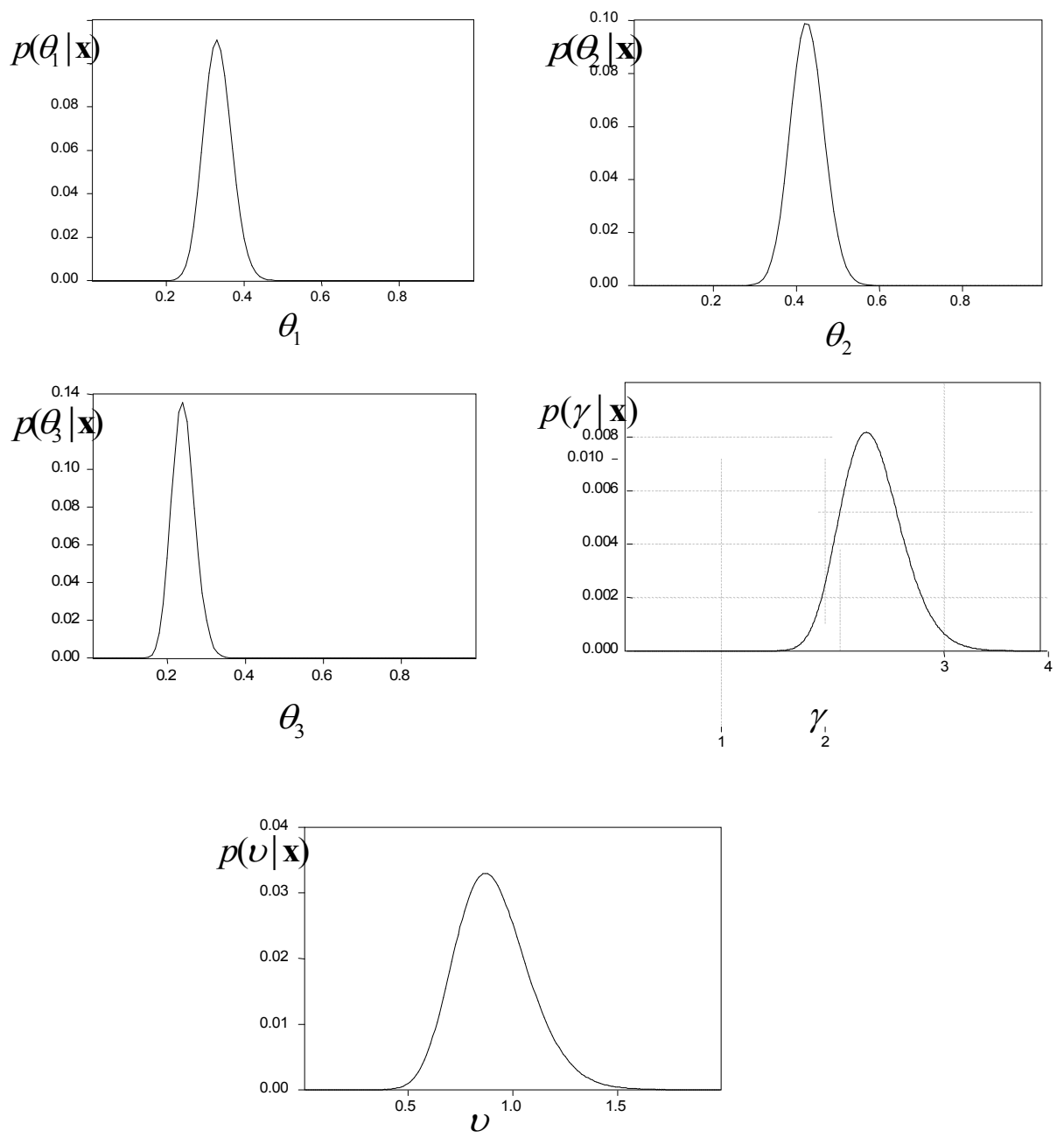

Fig. 3.1: The Marginal Posterior Distributions of the Parameters

The posterior means are achieved for the data set given in Table 3.1 by programming in the SAS package. The Bayesian estimates (means) of the parameters $\theta_{1}, \theta_{2}, \theta_{3}, \gamma$ and $v$ are obtained to be $0.3291,0.4484,0.2225$, 2.7445 and 1.73709 , respectively. The posterior modes are obtained in the SAS package by solving the following system of simultaneous equations:

$$
\begin{aligned}
& g_{i} / \theta_{i}-\sum_{j \neq i=1}^{m} r_{i j}\left(\gamma+v \theta_{j}\right) /\left(\gamma \theta_{i}+\theta_{j}+v \theta_{i} \theta_{j}\right)-\sum_{j \neq i=1}^{m} r_{j i}\left(1+v \theta_{j}\right) /\left(\theta_{i}+\gamma \theta_{j}+v \theta_{i} \theta_{j}\right)=0, \\
& \quad i=1,2, \ldots, m . \\
& T / v-\sum_{j \neq i=1}^{m} r_{i j}\left(\theta_{j} \theta_{i}\right) /\left(\gamma \theta_{i}+\theta_{j}+v \theta_{i} \theta_{j}\right)-\sum_{j \neq i=1}^{m} r_{j i}\left(\theta_{i} \theta_{j}\right) /\left(\theta_{i}+\gamma \theta_{j}+v \theta_{i} \theta_{j}\right)=0, \\
& K / \gamma-\sum_{j \neq i=1}^{m} r_{i j} \theta_{i} /\left(\gamma \theta_{i}+\theta_{j}+v \theta_{i} \theta_{j}\right)-\sum_{j \neq i=1}^{m} r_{j i} \theta_{j} /\left(\theta_{i}+\gamma \theta_{j}+v \theta_{i} \theta_{j}\right)=0,
\end{aligned}
$$




$$
\sum_{i=1}^{m} \theta_{i}-1=0
$$

The posterior modes of the parameters $\theta_{1}, \theta_{2}, \theta_{3}, \gamma$ and $v$ are found to be $0.3291,0.4477,0.2232,2.5816$ and 1.5793 , respectively. The results (posterior means and modes) imply that treatment $T_{2}$ is better than the other two treatments. The treatment $T_{1}$ is the next preferred one and $T_{3}$ is the least favored one.

To test the hypotheses: $H_{i j}: \theta_{i}>\theta_{j}$ and $H_{i j}{ }^{c}: \theta_{j} \geq \theta_{i}, i \neq j=1,2,3$, the posterior probability for $H_{i j}, p_{i j}=p\left(\theta_{i}>\theta_{j}\right)$ is attained using a transformation: $\varphi=\theta_{i}-\theta_{j}$ and $\xi=\theta_{i}$ so the posterior probability $p_{i j}$ is:

$$
p_{i j}=p(\varphi>0 \mid \mathbf{x})=\int_{\varphi=0}^{1} \int_{\xi=\varphi}^{(1+\varphi) / 2} \int_{\gamma=0}^{\infty} \int_{v=0}^{\infty} p(\varphi, \xi, \gamma, v \mid \mathbf{x}) d v d \gamma d \xi d \varphi .
$$

The program is run in the SAS package and the posterior probabilities $p_{i j}$ and $q_{i j}$ are obtained and given in the Table 3.2.

Table 3.2: Posterior Probabilities using Uniform Prior

\begin{tabular}{c|ccc}
\hline Hypotheses & $p_{i j}$ & Hypotheses & $q_{i j}$ \\
\hline$H_{12}: \theta_{1}>\theta_{2}$ & 0.0323 & $H_{12}^{c}: \theta_{2} \geq \theta_{1}$ & 0.9677 \\
\hline$H_{13}: \theta_{1}>\theta_{3}$ & 0.9386 & $H_{13}^{c}: \theta_{3}>\theta_{1}$ & 0.0614 \\
\hline$H_{23}: \theta_{2}>\theta_{3}$ & 0.9990 & $H_{23}^{c}: \theta_{3}>\theta_{2}$ & 0.0001 \\
\hline
\end{tabular}

The hypotheses $H_{12}^{c}$ and $H_{23}$ are accepted showing the preference of treatment $T_{2}$. For $H_{13}$, the evidence is conclusive. $H_{13}$ is accepted making evident treatment $T_{1}$ is considered to be preferred to $T_{3}$.

The predictive probability is the probability that treatment $T_{1}$ is preferred to treatment $T_{2}$ in the future single comparison of these two treatments. The predictive probabilities using uniform prior are attained with the help of programming in the SAS package.

Table 3.3: The Predictive Probabilities using Uniform Prior

\begin{tabular}{c|cccccc}
\hline Pairs $(i, j)$ & $(1,2)$ & $(2,1)$ & $(1,3)$ & $(3,1)$ & $(2,3)$ & $(3,2)$ \\
\hline$P_{i j}(1)$ & 0.5588 & 0.6748 & 0.7180 & 0.5689 & 0.7541 & 0.4923 \\
\hline$P_{i j}(2)$ & 0.2838 & 0.1855 & 0.1817 & 0.3137 & 0.1405 & 0.3692 \\
\hline$P_{i j}(0)$ & 0.1574 & 0.1396 & 0.1003 & 0.1175 & 0.1054 & 0.1385 \\
\hline
\end{tabular}


The effect of order of presentation is quite overwhelming. The predictive probabilities of no preference among all the pairwise comparisons of the treatments are less than 0.16 .

\section{Appropriateness of the Model}

The hypotheses to test the appropriateness of the model are:

$H_{o}$ : The model is considered to be true for any value of $\theta=\theta_{o}$.

$H^{c}$ : The model is considered not to be true for any value of $\theta$.

To test the appropriateness of the model in case of three treatments, observed number of preferences is compared with the expected number of preferences. The $\chi^{2}$ statistic is used to test the goodness of fit of the model for paired comparison. The $\chi^{2}$ Statistic is:

$$
\begin{array}{r}
\chi^{2}=\sum_{i \neq j}^{m}\left\{\frac{\left(w_{i j}(1)-\hat{w}_{i j}(1)\right)^{2}}{\hat{w}_{i j}(1)}+\frac{\left(w_{i j}(2)-\hat{w}_{i j}(2)\right)^{2}}{\hat{w}_{i j}(2)}+\frac{\left(t_{i j}-\hat{t}_{i j}\right)^{2}}{\hat{t}_{i j}}+\right. \\
\left.\frac{\left(w_{j i}(1)-\hat{w}_{j i}(1)\right)^{2}}{\hat{w}_{j i}(1)}+\frac{\left(w_{j i}(2)-\hat{w}_{j i}(2)\right)^{2}}{\hat{w}_{j i}(2)}+\frac{\left(t_{j i}-\hat{t}_{j i}\right)^{2}}{\hat{t}_{j i}}\right\}
\end{array},
$$

with $2 m(m-1)-(m+1)$ degree of freedom taken from 'and Davidson and Beaver (1977). Where $\hat{w}_{i j}(1)$ and $\hat{w}_{i j}(2)$ are the expected number of times $T_{i}$ and $T_{j}$ are preferred respectively and $\hat{t}_{i j}$ is the expected number of times $T_{i}$ and $T_{j}$ end up in a tie when $T_{i}$ is presented first. Similarly, $\hat{w}_{j i}(1), \hat{w}_{j i}(2)$ and $\hat{t}_{j i}$ are described when $T_{j}$ is presented first.

Table 4.1: Observed and Expected Number of Preferences

\begin{tabular}{c|cccccc}
\hline Pairs $(i, j)$ & $w_{i j}(1)$ & $\hat{w}_{i j}(1)$ & $w_{i j}(2)$ & $\hat{w}_{i j}(2)$ & $t_{i j}$ & $\hat{t}_{i j}$ \\
\hline$(1,2)$ & 23 & 23.59 & 11 & 11.71 & 8 & 6.70 \\
$(2,1)$ & 29 & 29.14 & 6 & 7.79 & 8 & 6.07 \\
$(1,3)$ & 27 & 30.99 & 11 & 7.64 & 5 & 4.37 \\
$(3,1)$ & 22 & 24.04 & 14 & 12.95 & 6 & 5.00 \\
$(2,3)$ & 34 & 31.02 & 6 & 5.61 & 1 & 4.37 \\
$(3,2)$ & 23 & 20.82 & 16 & 15.28 & 3 & 5.90 \\
\hline
\end{tabular}

The value of the $\chi^{2}$ statistic is obtained as 8.48 and the $p$-value is 0.39 which interprets that the model is suitable for the data.

\section{Conclusion}

Bayesian analysis of a paired comparison model, the van Baaren Model VI, has proved the model to be appropriate to fit. Using the noninformative uniform prior, both the posterior estimates (means and modes) have depicted similar values. When the treatment parameters are compared via Bayesian testing of hypotheses, it is evident that the treatment $T_{2}$ is the most favoured one. This 
result is also in accord with the ranking obtained by the posterior estimates. The preference of any of the treatment over any other one in a future single comparison cannot be predicted because of the overwhelming order effect. Thus the said model is interpreted as appropriate for the data and is useful for the cases of sensory paired comparison testing.

\section{References}

1. Aslam, M. (2002). Bayesian Analysis for Paired Comparisons Models Allowing Ties and Not Allowing Ties. Pak. J. Statist., Vol.18, 1, 53-69.

2. Aslam, M. (2003). An Application of Prior Predictive Distribution to Elicit the Prior Density. Journal of Statistical Theory and Applications, Vol.2, 1, 70-83.

3. Aslam, M. (2005). Bayesian Comparison of the Paired Comparison Models Allowing Ties. Journal of Statistical Theory and Applications, Vol.4, 2, 161-172.

4. Baker, S., Jennings, P., Dunne, G., and Williams, R. (2004). Improving the Effectiveness of Paired Comparison Tests for Automotive Sound Quality. Eleventh International Congress on Sound and Vibration.5-8 July 2004.St. Petersburg, Russia.

5. Beaver, R.J., and Gokhale, D.V. (1975). A Model to Incorporate WithinPair Order Effects in Paired Comparisons. Comm. Statist., 4 (10), 923939.

6. Bradley, R. A., and Terry. M.E. (1952). Rank Analysis of Incomplete Block Design:I. The Method of Paired Comparisons. Biometrika,39, 324-345.

7. Bradley, R.A. (1953). Some Statistical Methods in Taste Testing and Quality Evaluation. Biometrics,9, 22-38.

8. Davidson, R.R. (1970). On Extending the Bradley-Terry Model to Accommodate Ties in Paired Comparison Experiments. Journal of American Statistical Association, Vol. 65, 329, 317-28.

9. Davidson, R.R. and Beaver, R.J. (1977). On Extending the Bradley-Terry Model to Incorporate within Pair Order Effects. Biometrics,33, 4, 693-702.

10. Davidson, R.R. and Solomon, D.L. (1973). A Bayesian Approach to Paired Comparison Experimentation. Biometrika, 60, 3, 477-87.

11. Glickman, M.E. (2008). Bayesian Locally Optimal Design of Knockout Tournaments. Journal of Statistical Planning and Inference, 138, 2117-2127.

12. Henry, R.J. (1992). An Extension to the Thurstone- Mosteller Model for Chess. The Statistician, 41, 559-567.

13. Kim, D.H., and Kim, H.J. (2004). A Bayesian Approach to Paired Comparison of Several Products of Poisson Rates. Proceedings of the Autumn Conference, Korean Statistical Society.

14. Kim, H.J. (2005). A Bayesian Approach to Paired Comparison rankings Based on a Graphical Model. Computational Statistics \& Data Analysis, Vol. 48, 2, 269-290. 
15. Laplace, P.S. (1812). Thkorie Analytique des Probabilitks, Paris: Courcier.

16. Li, L., and Kim, K. (2000). Estimating Driver's Crash Risks Based on Extended Bradley-Terry Model: An Induced Exposure Method. Jr. R. Statist. Soc. A, Vol.163, 2, 227-240.

17. Luce, R.D. (1959). Individual Choice Behavior: A Theoretical Analysis. John Wiley \& Sons, New York.

18. Marcus, D.J. (2000). Focus on Sport. New Table-Tennis Rating System. The Statistician, Vol. 50, 2, 191-208.

19. Marrin, M.L., Mclntosh, K.A., Keane, D., and Schmuck, M.L. (2004). Use of the Paired-Comparison Technique to Determine the Most Valued Qualities of the McMaster Medical Programme Admissions Process. Advances in Health Sciences Education Theory and Practice, 9, 129-135. Springer Publishers.

20. Mazzuchi, T.A., William, G.L., and Bruning, A. (2008). A Paired Comparison Experiment for Gathering Expert Judgment for an Aircraft Wiring Risk Assessment. Reliability Engineering and System Safety, 93, 722-731.

21. Neuman, K., and Watson, B.G. (1993). Application of Paired Comparison Methodology in Measuring Canadian's Forest Values. Proceedings of the Forty-Eighth Annual Conference of the American Association for Public Opinion Research. The Public Opinion Quarterly, Vol. 57, 3, 437-464.

22. Rao, P.V., and Kupper, L.L. (1967). Ties in Paired Comparison Experiments: A Generalization of the Bradley-Terry Model. Journal of the American Statistical Association, Vol. 62, 317, 194-204.

23. Simons, T.R., Shriner, S. A. and Farnsworth, G. L. (2006). Comparison of Breeding Bird and Vegetation Communities in Primary and Secondary Forests of Great Smoky Mountains National Park. Biological Conservation, 129, 302-311.

24. Stern, H. (1990). A Continuum of Paired Comparison Models. Biometrika, $77,2,265-273$.

25. Thurstone, L.L. (1927). A Law of Comparative Judgment. Psychological Review, 34:273-286.

26. van Baaren, A. (1978). On a Class of Extensions to the Bradley-Terry Model in Paired Comparisons. Statistica Neerlandica, Vol. 32, 57-66.

27. Watson, A.B. (2001). Subjective Estimation of Visual Quality. Research and Technology Report.

28. Zermelo, E. (1929). Die Berechnung der Turnier-Ergebnissealsein Maximumproblem der Warscheinlichkeitsrechnung. Math. Zeit., 29, 436-460. 